\title{
P20-20 LB. Indels in V4 share sequence features with insertions and deletions during somatic hypermutation of immunoglobulins S Guglietta*, G Pantaleo and C Graziosi
}

Address: CHUV, Lausanne, Switzerland

* Corresponding author

from AIDS Vaccine 2009

Paris, France. 19-22 October 2009

Published: 22 October 2009

Retrovirology 2009, 6(Suppl 3):P424 doi:10.1 I86/I742-4690-6-S3-P424

This abstract is available from: http://www.retrovirology.com/content/6/S3/P424

(C) 2009 Guglietta et al; licensee BioMed Central Ltd.

\section{Background}

We have shown that gp120 V4 is characterized by indels multiples of three base pairs and with no generation of stop codons. Similar insertions and deletions have also been reported during somatic hypermutation (SHM) of immunoglobulins. In this study, we have compared sequence patterns associated to indels in V4 to those reported in immunoglobulins. Our aims were i) to assess similarities between indels in V4 and SHM; ii) to assess whether sequence features of indels in V4 could also be identified in V3 and C3.

\section{Methods}

RT-PCR amplification of an Env fragment spanning C2C4 derived from HIV plasma RNA from 12 patients at early stage disease.

\section{Results}

In the $\mathrm{N}$-terminal end of V4, indels involved duplications of a 15 mer and a 9 mer, designated as SeqA (consensus ACA ACA AAA CTG TTT) and SeqB (consensus AGT ACT TGG), respectively. In the C-terminal end, indels involved homonulceotide runs, AAT stretches and other non-specific sequences. Trinucleotides, repeats and palindromes such as those described in SHM were recognized in V4 in all the patients analyzed. No duplications analogous to SeqA and SeqB could be recognized in V3 and C3 in all the patients analyzed. Trinucleotides and palindromes were detected in both regions.

\section{Conclusion}

Indels in V4 are associated to sequence features similar to those described during SHM of immunoglobulins. The presence of structural intermediates consisting of repeats, duplications, and/or palindromes indicates that indels may be the product of a mechanism of DNA misalignment, with indels resulting from DNA strand slippage during DNA synthesis. A major consequence of this mechanism is that mutations in the gp120 regions analyzed are not random but appear to be directed by the presence of sequence motifs in the nearby DNA. Further studies are needed to elucidate the mechanism of indels in hypervariable regions of gp 120. 